\title{
The Incidence of Adjacent Segment Degeneration after the Use of a Versatile Dynamic Hybrid Stabilization Device in Lumbar Stenosis: Results of a 5-8-Year Follow-up
}

\author{
Mauro Dobran, Davide Nasi, Domenico Paolo Esposito, Maurizio Gladi, \\ Massimo Scerrati, Maurizio Iacoangeli \\ Department of Neurosurgery, Umberto I University General Hospital, Università Politecnica delle Marche, Ancona, Italy
}

\begin{abstract}
Study Design: Retrospective study with long-term follow-up.
Purpose: To evaluate the long-term incidence of adjacent segment degeneration (ASD) and clinical outcomes in a consecutive series of patients who underwent spinal decompression associated with dynamic or hybrid stabilization with a Flex+TM stabilization system (SpineVision, Antony, France) for lumbar spinal stenosis.

Overview of Literature: The incidence of ASD and clinical outcomes following dynamic or hybrid stabilization with the Flex+TM system used for lumbar spinal stenosis have not been well investigated.

Methods: Twenty-one patients with lumbar stenosis and probable post-decompressive spinal instability underwent decompressive laminectomy followed by spinal stabilization using the Flex+TM stabilization system. The indication for a mono-level dynamic stabilization was a preoperative magnetic resonance imaging (MRI) demonstrating evidence of severe disc disease associated with severe spinal stenosis. The hybrid stabilization (rigid-dynamic) system was used for multilevel laminectomies with associated initial degenerative scoliosis, first-grade spondylolisthesis, or rostral pathology.

Results: The improvement in Visual Analog Scale and Oswestry Disability Index scores at follow-up were statistically significant $(p<0.0001$ and $p<0.0001$, respectively). At the 5-8-year follow-up, clinical examination, MRI, and $\mathrm{X}$-ray findings showed an ASD complication with pain and disability in one of 21 patients. The clinical outcomes were similar in patients treated with dynamic or hybrid fixation.

Conclusions: Patients treated with laminectomy and Flex+TM stabilization presented a satisfactory clinical outcome after 5-8 years of follow-up, and ASD incidence in our series was $4.76 \%$ (one patient out of 21). We are aware that this is a small series, but our long-term follow-up may be sufficient to contribute to the expanding body of literature on the development of symptomatic ASD associated with dynamic or hybrid fixation.
\end{abstract}

Keywords: Adjacent segment degeneration; Dynamic stabilization; Hybrid stabilization; Lumbar stenosis

Received Jul 23, 2017; Revised Sep 7, 2017; Accepted Sep 11, 2017

Corresponding author : Davide Nasi

Department of Neurosurgery, Umberto I General Hospital, Università Politecnica delle Marche, Via Conca \#71, Ancona, 60020, Italy

Tel: +39-0715964567, Fax: +39-0715964575, E-mail: davidenasi83@gmail.com 


\section{Introduction}

For many decades, lumbar fusion surgery has been considered the gold standard surgical technique for patients suffering from chronic lower back pain caused due to degenerative disease and instability. Although favorable clinical outcomes can be achieved, complications of lumbar fusion with rigid fixation have gained increasing attention [1]. For many researchers, fusion is one of the risk factors for adjacent segment disc degeneration, with an incidence rate of $14 \%-70 \%$ of operated patients [2-4]. Currently, many dynamic devices are available for lumbar spine dynamic stabilization, and each has specific technologies which attempt to preserve the physiological range of motion of the lumbar spine [2-7]. Adjacent segment degeneration (ASD) may be caused due to altered biomechanics of the fused spine producing abnormal forces on the adjacent spinal levels with degeneration of the rostral disc adjacent to a rigid stabilization [8]. ASD is defined as new degenerative changes at the spinal level, adjacent to surgically treated level or levels in the spine, accompanied by related symptoms (radiculopathy, myelopathy, or instability). The instability is assessed using dynamic X-ray examination. Disk degeneration disease represents the radiographical changes without symptoms [9].

Posterior dynamic stabilization could ensure a relatively normal range of motion of the instrumented segments, thus avoiding the rapid degeneration of the adjacent intervertebral disc [10-12]. Dynamic stabilization is indicated in cases of degenerative disc disease or may be used in patients with lumbar spinal stenosis treated with extensive laminectomy to prevent long-term spinal instability, especially when preoperative magnetic resonance imaging (MRI) shows pathological disc changes at the same or adjacent level to the planned laminectomy $[9,13,14]$. This study was performed to evaluate the clinical outcomes and incidence of ASD syndrome 5-8 years after performing extensive laminectomy and dynamic or hybrid stabilization utilizing the Flex+TM system for treating lumbar spinal stenosis (SpineVision, Antony, France).

\section{Materials and Methods}

The Flex+TM device is a rod that can be used with pedicle screws. It is made of rigid titanium alloy (TA6V) end pieces combined with a dynamic segment consisting of a twisted titanium alloy cable covered with a polycarbonate

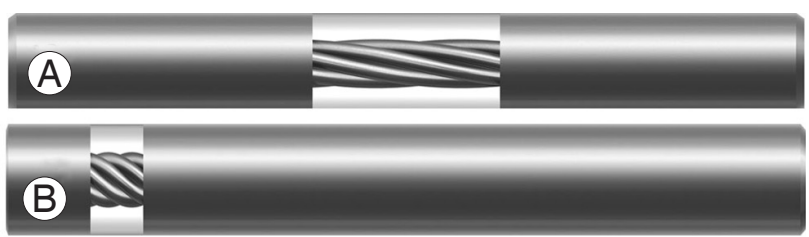

Fig. 1. (A, B) The Flex+TM device (SpineVision, Antony, France) is a rod that can be used with pedicle screws. It is made up of the rigid titanium alloy (TA6V) at extremities and a dynamic part consisting of a twisted titanium alloy cable over-molded with polycarbonate urethane polymer.

urethane polymer (Fig. 1A, B).

Twenty-one patients were included in this study from September 2008 to May 2011 (10 males and 11 females). The mean age at the time of surgery was 64.3 years (range, 49 to 77 years). All the patients who were affected by lumbar stenosis and suspected to have post-decompression instability were treated with wide laminectomy (including partial demolition of the facet joint) or posterior foraminotomy followed by stabilization utilizing Flex+TM (dynamic or hybrid construct, SpineVision). The indication for a one-level dynamic stabilization was a preoperative MRI providing evidence of a pathological disc (Pfirmann 2-3-4) at the same level as that of the planned laminectomy. The hybrid device (one-level dynamic stabilization and multilevel rigid fixation) was used in cases of a multilevel laminectomy with associated initial degenerative scoliosis (Schwab classification VB0), first-grade spondylolisthesis, or a rostral pathological disc [15,16] (Fig. $2 \mathrm{~A}-\mathrm{C})$. None of the patients had undergone any previous spinal surgery. The dynamic device was used in $12 \mathrm{pa}-$ tients (group A) and hybrid device in nine (four patients at two levels, five patients at three or more levels; group B). All operated patients had disabling low back pain not responsive to a conservative treatment continued for at least 6 months prior to their surgical procedure. Patients with infections, tumors, severe scoliosis and spondylolisthesis, diabetes and, metabolic diseases were excluded from this study. Preoperative clinical and radiological evaluation consisted of a neurological examination, Visual Analog Scale (VAS) and Oswestry Disability Index (ODI), preoperative MRI, and dynamic X-ray. Follow-up visits, including clinical and radiological assessments, were conducted at 3,12, and 24 months (mean follow-up, 12 months) following discharge (Fig. 3A) and final control at 5-8 years after surgery. All operations were performed under general anesthesia with the patient in a neutral and prone 

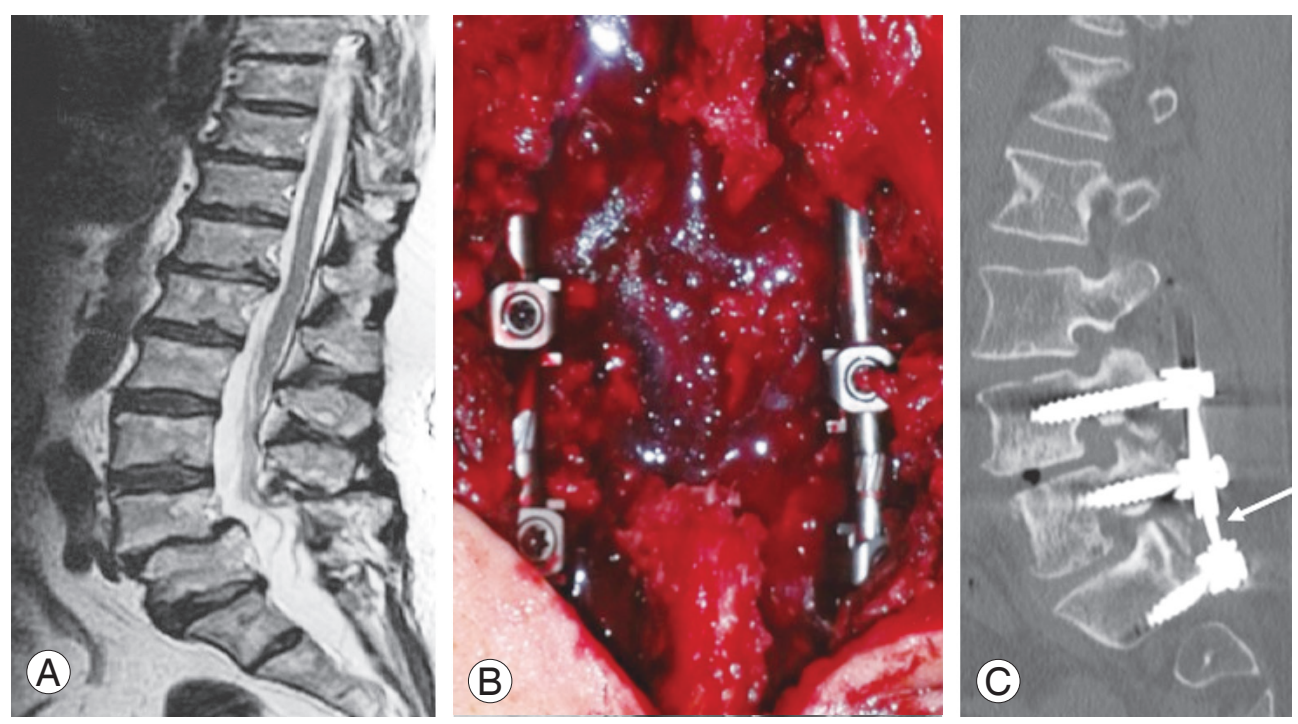

Fig. 2. Pre-, intra-, and postoperative imaging of patient 21 of Table 1. This patient was a 74-year-old female with 6 months' history of $L 5$ bilateral radiculopathy and claudication. (A) Preoperative sagittal T2-weighted magnetic resonance imaging showing L4-S1 stenosis with the L4-L5 pathological disc (Pfirmann 4) at the same level as that of the planned laminectomy with first-grade spondylolisthesis and a caudal pathological disc (Pfirmann 4). (B) Intraoperative picture of a three-level stabilization (L4-L5-S1) with the hybrid device (rigid L4-L5 and dynamic L5-S1). (C) Postoperative computed tomography with sagittal reconstruction demonstrating the correct positioning of the device and dynamic segment of stabilization (white arrow).
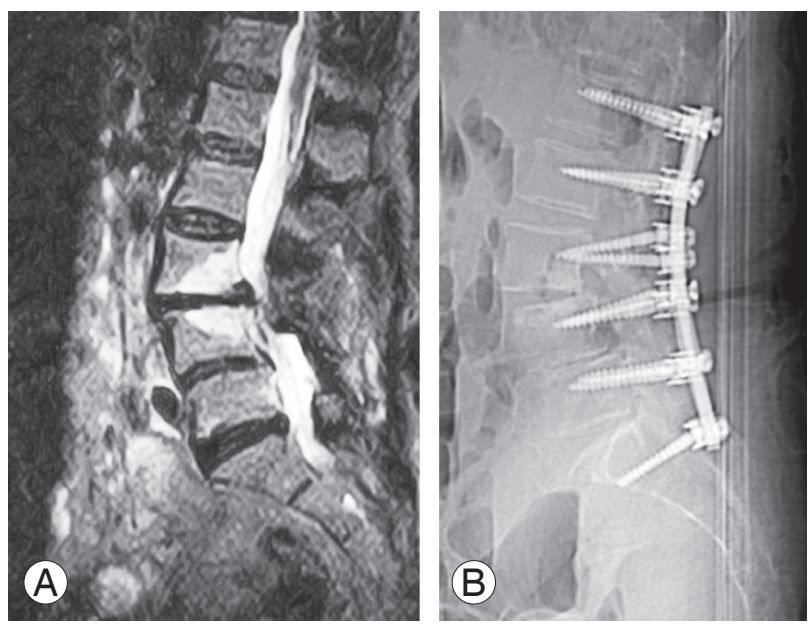

Fig. 3. (A) After 5 years, the patient presented a new onset of radiculopathy and claudication, and magnetic resonance imaging showed an adjacent segment degeneration with stenosis one level above the stabilization. (B) Postoperative X-ray. The patient was re-operated with extension of laminectomy on the above levels and six rigid level stabilization (L1-S1).

position; the surgical approach was along the midline, and the extension of laminectomy was performed according to the clinical data. Screws (PLUS, X-PLUS pedicular screws; SpineVision) were placed under fluoroscopic visualization. Statistical analyses were performed using the Mann-
Whitney test and Student $t$-test. All patients granted permission for this study before surgery. All preoperative patient data are summarized in Table 1.

All procedures performed in studies involving human participants were in accordance with the ethical standards of the institutional and/or national research committee and with the 1964 Helsinki Declaration and its later amendments or comparable ethical standards. The institutional review board or similar entity approval was not necessary for this study. The risk to participants was minimal. The research data analysis did not affect the participants and their medical care. Informed consent was obtained from all individual participants included in the study.

\section{Results}

At admission, the mean ODI and VAS score were $40.1 \%$ and 7.2, respectively. At the 6-month follow-up, the mean ODI and VAS score were $12.7 \%$ and 2.2 , respectively; these differences were statistically significant $(p<0.0001$ and $p<0.0001$ ) (Table 2). The mean ODI and VAS score improvement in group A was $23.2 \%$ and 4.8 , respectively ( $p=0.0005$ and $p=0.0001$ ) (Table 3$)$. The postoperative sixmonth VAS score modification $(p=0.0006)$ as well as the 


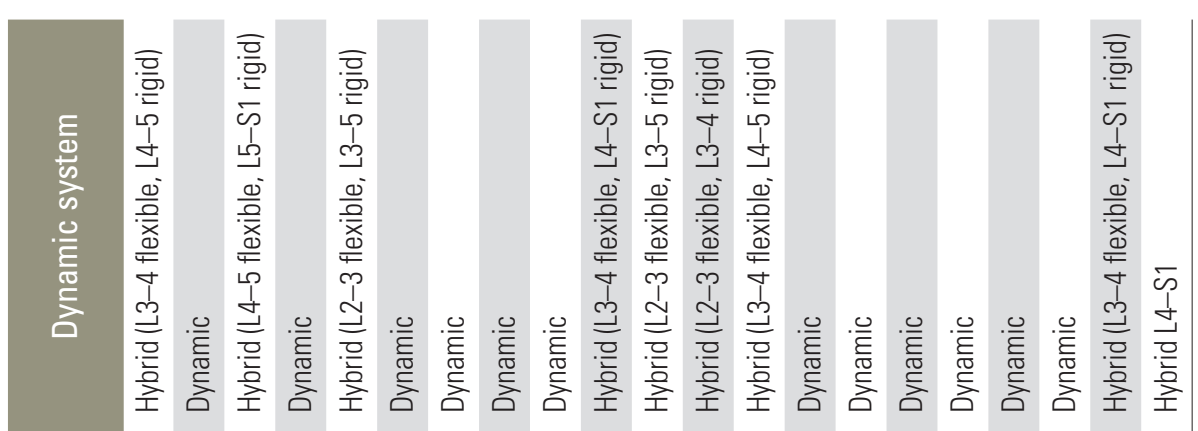

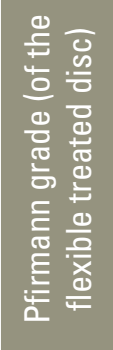

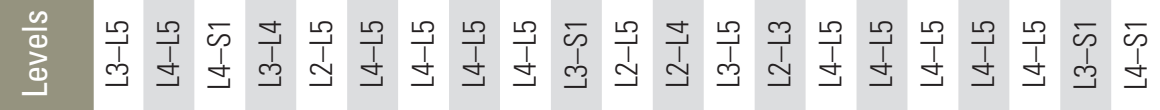

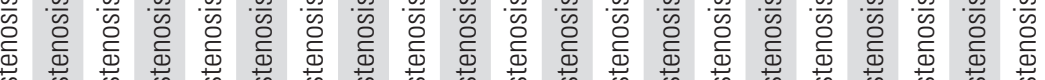
क क क

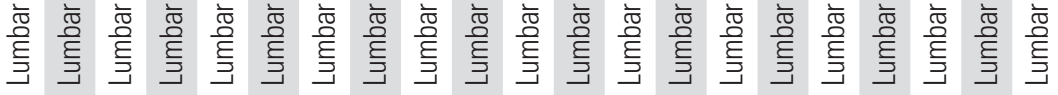

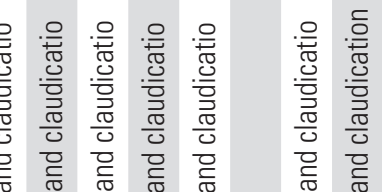

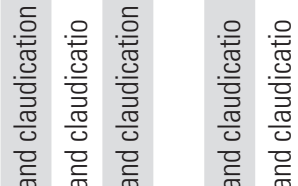

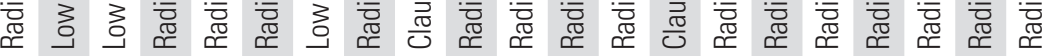

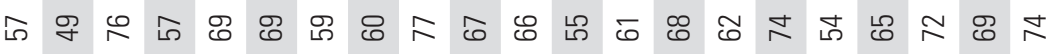
н 


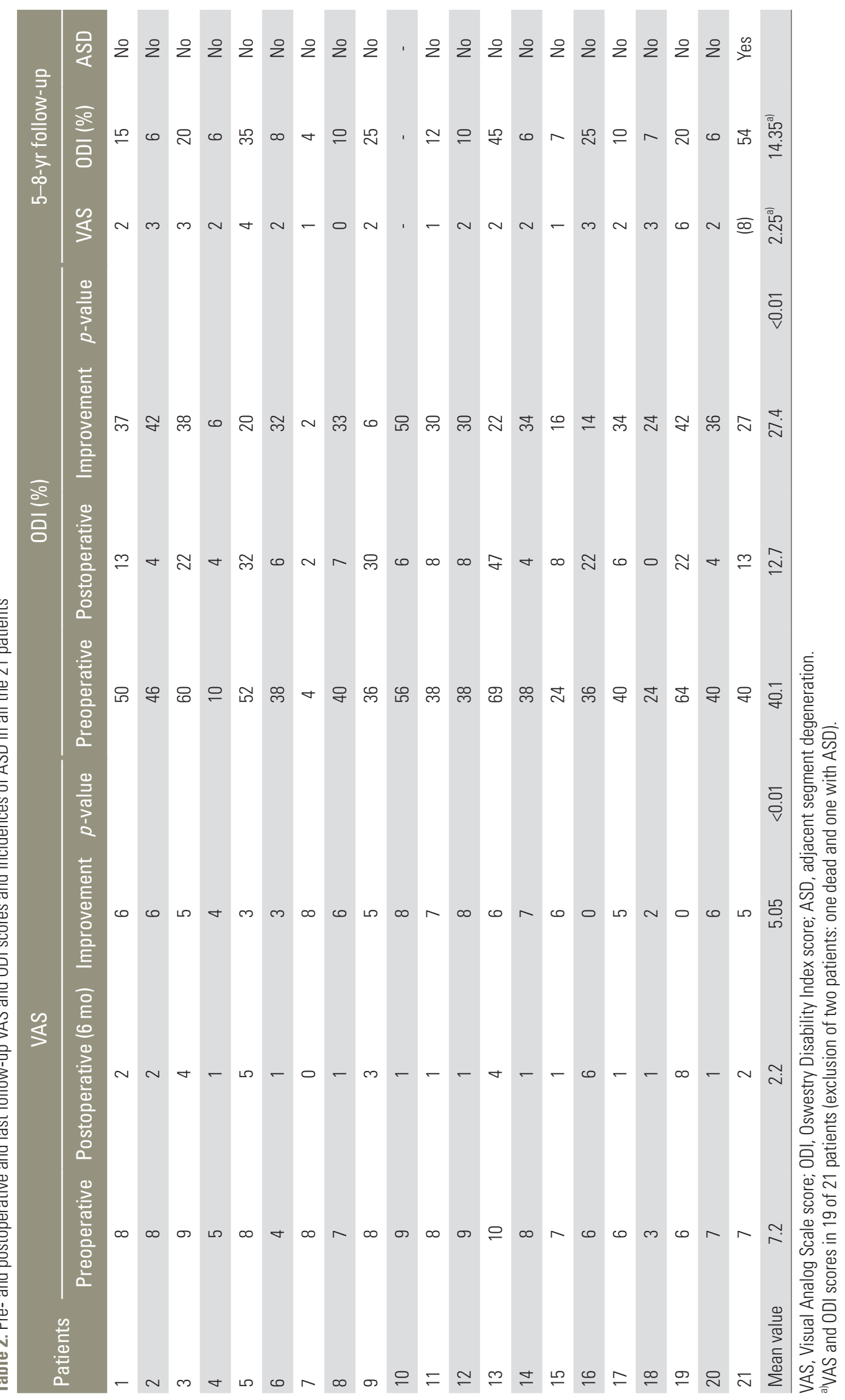


Table 3. Pre- and postoperative VAS and ODI scores in group A (dynamic device)

\begin{tabular}{|c|c|c|c|c|c|c|c|}
\hline \multirow[b]{2}{*}{ Patients } & \multicolumn{3}{|c|}{ VAS } & \multicolumn{4}{|c|}{ ODI (\%) } \\
\hline & Preoperative & $\begin{array}{l}\text { Postoperative } \\
\text { (6 mo) }\end{array}$ & Improvement $p$-value & Preoperative & Postoperative & Improvement & $p$-value \\
\hline 1 & 8 & 2 & 6 & 46 & 4 & 42 & \\
\hline 2 & 5 & 1 & 4 & 10 & 4 & 6 & \\
\hline 3 & 4 & 1 & 3 & 38 & 6 & 32 & \\
\hline 4 & 8 & 0 & 8 & 4 & 2 & 2 & \\
\hline 5 & 7 & 1 & 6 & 40 & 7 & 33 & \\
\hline 6 & 8 & 3 & 5 & 36 & 30 & 6 & \\
\hline 7 & 8 & 1 & 7 & 38 & 4 & 34 & \\
\hline 8 & 7 & 1 & 6 & 24 & 8 & 16 & \\
\hline 9 & 6 & 6 & 0 & 36 & 22 & 14 & \\
\hline 10 & 6 & 1 & 5 & 40 & 6 & 34 & \\
\hline 11 & 3 & 1 & 2 & 24 & 0 & 24 & \\
\hline 12 & 7 & 1 & 6 & 40 & 4 & 36 & \\
\hline Mean value & 6.4 & 1.5 & $<0.01$ & 31.3 & 8.08 & 23.2 & $<0.01$ \\
\hline
\end{tabular}

VAS, Visual Analog Scale score; ODI, Oswestry Disability Index score.

Table 4. Pre- and postoperative VAS and ODI scores in group B (hybrid device)

\begin{tabular}{|c|c|c|c|c|c|c|c|}
\hline \multirow{2}{*}{ Patients } & \multicolumn{3}{|c|}{ VAS } & \multicolumn{4}{|c|}{ ODI (\%) } \\
\hline & Preoperative & $\begin{array}{l}\text { Postoperative } \\
\text { (6 mo) }\end{array}$ & Improvement $p$-value & Preoperative & Postoperative & Improvement & $p$-value \\
\hline 1 & 8 & 2 & 6 & 50 & 13 & 37 & \\
\hline 2 & 9 & 4 & 5 & 60 & 22 & 38 & \\
\hline 3 & 8 & 5 & 3 & 52 & 32 & 20 & \\
\hline 4 & 9 & 1 & 8 & 56 & 6 & 50 & \\
\hline 5 & 8 & 1 & 7 & 38 & 8 & 30 & \\
\hline 6 & 9 & 1 & 8 & 38 & 8 & 30 & \\
\hline 7 & 10 & 4 & 6 & 69 & 47 & 22 & \\
\hline 8 & 6 & 8 & 0 & 64 & 22 & 42 & \\
\hline 9 & 8 & 3 & 5 & 53 & 20 & 33 & \\
\hline Mean value & 8.3 & 3.2 & $<0.01$ & 53.3 & 19.7 & 33.6 & $<0.01$ \\
\hline
\end{tabular}

VAS, Visual Analog Scale score; ODI, Oswestry Disability Index score.

ODI score $(p=0.0003)$ in group B were statistically significant. In this group, the mean ODI and VAS score improvement were $33.6 \%$ and 5.3 (Table 4). Matching the patients with a preoperative ODI score of $>40 \%$ and $<40 \%$, we obtained a greater improvement in the first group with a mean variation of $34.3 \%$ and $19.4 \%$, respectively $(p=0.0068)$. No significant differences were observed between the VAS and ODI score variations between patients treated with dynamic or hybrid device $(p=0.4636$ and $p=0.1325$ ).

There were two complications (one dural tear and one infection) which required a second operation. In the in- 
fected patient, the debridement of infected tissue did not require the removal of screw fixation as reported in the literature [6]. At the 5-8-year follow-up, clinical examination, MRI, and X-ray controls showed an ASD complication with pain and disability in one of 21 patients. Thus, clinical and MRI incidence of ASD in this study was $4.76 \%$.

In the remaining 20 patients, the mean VAS score was 2.2 and the mean ODI score was $14.3 \%$, which was consistent with a good outcome. The patient requiring repeated operation was not included in the statistical analysis. The patient with ASD had a three-level stabilization (L4-L5S1) with the hybrid device (rigid L4-L5, dynamic L5-S1) (Fig. 3A, B)and underwent an extension of laminectomy to the above levels and six-level rigid stabilization (L1-S1) 5 years after the original operation. No instrumentation failure occurred in this series to date.

\section{Discussion}

ASD following lumbar fusion may result in a variety of complications, especially in case of severe back pain not responsive to conservative therapy $[4,17,18]$. This condition, at least from a radiological point of view, is quite often related to a failed back spinal surgery. ASD remains a debatable issue because many researchers are uncertain if it is the natural evolution of an aging spine or is related to a previous fusion [1-23]. However, reports in the literature demonstrate the occurrence of clinical ASD in approximately $30 \%$ of operated patients. Age of the patient and a long segment fixation may be predisposing factors $[19,23]$. The etiology of ASD is likely to be multifactorial, involving the supporting musculature, external loads, and a combination of forces. We must remember that patients requiring an operation for segmental degeneration may have a higher risk of further degeneration than those not requiring surgery. However, a contributing factor in ASD may be a rigid spinal fixation system $[12,13]$. The length of the fusion has been found to be related to the occurrence of ASD, ranging from $32 \%$ for a one-level fusion to $66 \%$ for three or more levels [14].

Over the past 20 years, many lumbar dynamic devices have been introduced to reduce the incidence of ASD. In the present series, we used the same dynamic devices in all patients (Flex+TM system, SpineVision) to stabilize a single spinal segment while the hybrid device was used to treat two or more segments to prevent the evolution of the degenerative disc disease and the development of a post-laminectomy instability. Moreover, the hybrid device seems to be useful in patients who have undergone a multilevel laminectomy to grant stability at the decompressed levels and protection to the adjacent disc segment. Dynamic stabilization seems to achieve satisfactory clinical outcomes even in cases presenting with disabling preoperative pain. This was evident from the considerable ODI score improvement in patients with preoperative ODI scores $>40 \%$. The clinical improvement of patients treated with dynamic versus hybrid devices was not statistically significant, indicating that both the types of constructs are effective. We had no complications related to the implants or materials used in the system. Thus far, there have been no reports of pedicle screw loosening in patients with dynamic implants [1-23]. In our patients, the long-term follow-up was important to indicate a true rate of subsequent symptoms requiring reoperation for ASD because it was observed in only one of the 21 operated patients.

In several studies, the prevalence of ASD and reoperation rates were higher in lumbar fusion procedures than in motion preservation procedures.

A recent meta-analysis revealed that the prevalence of ASD in the fusion group was 14.4 whereas that in the motion preservation group was $5.1 \%$ [22]; the reoperation rate in the fusion group was significantly higher than that in the motion preservation group. In their meta-analysis, several dynamic devices were included other than the Flex+TM system. The present study confirmed these data with the use of the Flex+TM device (SpineVision) during a long-term follow-up period. In fact, in our series, the reoperation rate for symptomatic ASD was $4.76 \%$ (one patient out of 21). This could be explained by the fact that the motion preservation device protects the spine from mechanical overload imposed by a rigid fixation of the spine while retaining spine movement and preventing ASD.

The Flex+TM device (SpineVision) is a recently available dynamic and hybrid stabilization option that may be useful in preventing post-laminectomy instability as well as adjacent disc degeneration. In the present series, we used the dynamic device for single segment stabilization to protect the involved disc against further degeneration. In cases of multilevel laminectomies with an associated initial degenerative scoliosis (Schwab classification VB0), first-grade spondylolisthesis, or an adjacent pathological disc, a hybrid device was implanted to stabilize the decompressed level, protect the adjacent disc with the dy- 
namic segment of the rod, and avoid late spinal instability of the rigid segment. From our experience, the Flex+TM device (SpineVision) is a novel dynamic and hybrid stabilization option that may be useful in preventing post-laminectomy instability as well as adjacent disc degeneration. Finally, the combined use of dynamic and rigid systems in many patients of this series minimized their biomechanical differences.

\section{Conclusions}

Patients treated with laminectomy and Flex+TM stabilization presented a satisfactory clinical outcome after a 5-8-year follow-up period, and the ASD incidence in this study was $4.76 \%$. We are aware that this is a small series, but our long-term follow-up period may be sufficient to contribute to the expanding body of literature on the development of symptomatic ASD associated with dynamic or hybrid fixation.

\section{Conflict of Interest}

No authors have any conflict of interest.

\section{References}

1. Cheh G, Bridwell KH, Lenke LG, et al. Adjacent segment disease followinglumbar/thoracolumbar fusion with pedicle screw instrumentation: a minimum 5-year follow-up. Spine (Phila Pa 1976) 2007;32:22537.

2. Dobran M, Nasi D, Brunozzi D, et al. Treatment of unstable thoracolumbar junction fractures: shortsegment pedicle fixation with inclusion of the fracture level versus long-segment instrumentation. Acta Neurochir (Wien) 2016;158:1883-9.

3. Kim $\mathrm{CH}$, Chung CK, Jahng TA. Comparisons of outcomes after single or multilevel dynamic stabilization: effects on adjacent segment. J Spinal Disord Tech 2011;24:60-7.

4. Wu JC, Huang WC, Tsai HW, et al. Pedicle screw loosening in dynamic stabilization: incidence, risk, and outcome in 126 patients. Neurosurg Focus 2011;31:E9.

5. Hasegawa K, Shimoda H, Kitahara K, Sasaki K, Homma T. What are the reliable radiological indicators of lumbar segmental instability? J Bone Joint
Surg Br 2011;93:650-7.

6. Ko CC, Tsai HW, Huang WC, et al. Screw loosening in the Dynesys stabilization system: radiographic evidence and effect on outcomes. Neurosurg Focus 2010;28:E10.

7. Kocak T, Cakir B, Reichel H, Mattes T. Screw loosening after posterior dynamic stabilization: review of the literature. Acta Chir Orthop Traumatol Cech 2010;77:134-9.

8. Bastian L, Lange U, Knop C, Tusch G, Blauth M. Evaluation of the mobility of adjacent segments after posterior thoracolumbar fixation: a biomechanical study. Eur Spine J 2001;10:295-300.

9. Chou WY, Hsu CJ, Chang WN, Wong CY. Adjacent segment degeneration after lumbar spinal posterolateral fusion with instrumentation in elderly patients. Arch Orthop Trauma Surg 2002;122:39-43.

10. Dobran M, Iacoangeli M, Di Somma LG, et al. Neurological outcome in a series of 58 patients operated for traumatic thoracolumbar spinal cord injuries. Surg Neurol Int 2014;5(Suppl 7):S329-32.

11. Pfirrmann CW, Metzdorf A, Zanetti M, Hodler J, Boos N. Magnetic resonance classification of lumbar intervertebral disc degeneration. Spine (Phila $\mathrm{Pa}$ 1976) 2001;26:1873-8.

12. Spallone A. A less-invasive technique for harvesting autologous iliac crest grafts for cervical interbody fusion: technical note. Surg Neurol 2007;67:160-2.

13. Putzier M, Hoff E, Tohtz S, Gross C, Perka C, Strube P. Dynamic stabilization adjacent to single-level fusion: part II. No clinical benefit for asymptomatic, initially degenerated adjacent segments after 6 years followup. Eur Spine J 2010;19:2181-9.

14. Tai CL, Hsieh PH, Chen WP, Chen LH, Chen WJ, Lai PL. Biomechanical comparison of lumbar spine instability between laminectomy and bilateral laminotomy for spinal stenosis syndrome: an experimental study in porcine model. BMC Musculoskelet Disord 2008;9:84.

15. Maserati MB, Tormenti MJ, Panczykowski DM, Bonfield CM, Gerszten PC. The use of a hybrid dynamic stabilization and fusion system in the lumbar spine: preliminary experience. Neurosurg Focus 2010;28:E2.

16. Tamburrelli FC, Proietti L, Logroscino CA. Critical analysis of lumbar interspinous devices failures: a retrospective study. Eur Spine J 2011;20 Suppl 1:S2735. 
17. Bono CM, Lee CK. Critical analysis of trends in fusion for degenerative disc disease over the past 20 years: influence of technique on fusion rate and clinical outcome. Spine (Phila Pa 1976) 2004;29:455-63.

18. Dobran M, Iacoangeli M, Nasi D, et al. Posterior titanium screw fixation without debridement of infected tissue for the treatment of thoracolumbar spontaneous pyogenic spondylodiscitis. Asian Spine J 2016;10:465-71.

19. Galbusera F, Bellini CM, Anasetti F, Ciavarro C, Lovi A, Brayda-Bruno M. Rigid and flexible spinal stabilization devices: a biomechanical comparison. Med Eng Phys 2011;33:490-6.

20. Morishita Y, Ohta H, Naito M, et al. Kinematic evaluation of the adjacent segments after lumbar instru- mented surgery: a comparison between rigid fusion and dynamic non-fusion stabilization. Eur Spine J 2011;20:1480-5.

21. Nakai S, Yoshizawa H, Kobayashi S. Long-term follow-up study of posterior lumbar interbody fusion. J Spinal Disord 1999;12:293-9.

22. Pan A, Hai Y, Yang J, Zhou L, Chen X, Guo H. Adjacent segment degeneration after lumbar spinal fusion compared with motion-preservation procedures: a meta-analysis. Eur Spine J 2016;25:1522-32.

23. Richolt J, Rauschmann M. Pedicle screw-based systems for dynamic stabilization: an insight into the philosophy, technique, indications and success of these systems. Orthopade 2010;39:602-8. 